\title{
N-Cadherin Nanoantagonist Driven Mesenchymal-to-Epithelial Transition in Fibroblasts for Improving Reprogramming Efficiency
}

Xia Meng ${ }^{1}$, Anwei Zhou ${ }^{2}$, Yu Huang ${ }^{3}$, Yu Zhang ${ }^{1}$, Yurui Xu*1, Kaifeng Shao*4 and Xinghai Ning*1

${ }^{1}$ National Laboratory of Solid State Microstructures, Collaborative Innovation Center of Advanced Microstructures, Chemistry and Biomedicine Innovation Center, College of Engineering and Applied Sciences, Jiangsu Key Laboratory of Artificial Functional Materials, Nanjing University, Nanjing, 210093, China

${ }^{2}$ National Laboratory of Solid State Microstructures, Collaborative Innovation Center of Advanced Microstructures, School of Physics, Nanjing University, Nanjing, 210093, China.

${ }^{3}$ West China School of Medicine, Sichuan University, Chengdu 610041, China.

${ }^{4}$ SARI Center for Stem Cell and Nanomedicine, Shanghai Advanced Research Institute, Chinese Academy of Sciences, Shanghai, 201210, China.

*Corresponding author

Email: dg1834024@smail.nju.edu.cn; shaokf1@gmail.com; xning@nju.edu.cn 


\section{Materials and Methods}

\section{Materials}

ADH-1 was purchased from MedchemExpress (New Jersey, USA). Tetrachloroauric acid $\left(\mathrm{HAuCl}_{4}\right)$ was purchased from Macklin (Shanghai, China). 3-(4,5-Dimethylthiazol-2-yl)-2,5-diphenyltetrazolium bromide (MTT) and Hoechst 33342 were purchased from Beyotime Institute of Biotechnology Co. LLC. (Nantong, China). SYBR Green PCR Master Mix was purchased from Yi Fei Xue Biotechnology (Nanjing, China). Alexa Fluor 594-conjugated goat anti-mouse secondary antibody, N-cadherin monoclonal antibody and Lipofectamine 2000 were purchased from Invitrogen Corporation (USA). N-cadherin-Fc chimeric protein and human IgG1 Fc fragment were purchased from R\&D Systems. Genomic DNA Purification Kit was purchased from Sangon biotech (Shanghai, China) and pMD18-T vector was purchased from Takara Bio USA, Inc.. Plat-E cells were purchased from Cyagen US Inc., and cultured in DMEM (Hyclone) supplemented with 10\% FBS (Gibco) and 1\% nonessential amino acids (NEAA) (Gibco). iPS cells were cultured in ES cell media (DMEM supplemented with 15\% FBS, penicillin/streptomycin, NEAA, $\beta$-mercaptoethanol, and with $1000 \mathrm{U} / \mathrm{ml}$ leukemia inhibitory factor (LIF)). All other chemicals and reagents were purchased from Sigma and Aladdin Reagent Company without further purification. Oct4-GFP transgenic allele-carrying mice were obtained from the Jackson Laboratory. C57 mice were purchased from Nanjing Qinglongshan Experimental Animal Center (Nanjing, China). All procedures were approved by the Medical Ethics Committee of the Affiliated Gulou Hospital of Nanjing University (SYXK(Su)2019-0056, Nanjing, China), and carried out in accordance with the National Institute of Health Guide for the Care and Use of Laboratory Animals.

\section{Measurement}

MTT assays were performed on a microplate reader (Tecan Group). The fluorescence of SSEA1 was measured by fluorescence microscopy (Nikon Instruments Inc.). The physical properties of ADH-AuNPs including the particle size distribution and zeta potential were measured using Zetasizer Nano ZS (Malvern). The morphology of 
ADH-AuNPs was measured by scanning electron microscope (SEM) (ZEISS ULTRA-55).

\section{Cell culture}

MEFs were isolated from e13.5 embryos hemizygous for the Oct4-GFP transgenic allele. In detail, head, internal organs, limbs and tails of the embryos were removed, the remaining tissues were manually sliced into small pieces and dissociated by the digestive solution $\left(1 \mathrm{mg} / \mathrm{mL}\right.$ collagenase IV) for $30 \mathrm{~min}$ at $37^{\circ} \mathrm{C}$ to obtain a single cell suspension. The cells were plated onto 10-cm culture dish in DMEM supplemented with $10 \%$ FBS and 1\% NEAA.

\section{Retroviral Infection}

The day before transduction, Plat-E cells were seeded at $4 \times 10^{6}$ cells per $100 \mathrm{~mm}$ culture dish. On the next day, pMXs-based retroviral vectors were transfected into Plat-E cells using Lipofectamine 2000 according to the manufacturer's instructions. After 12-h transduction, the medium was replaced. MEFs $\left(4 \times 10^{4}\right.$ cells $/$ well $)$ were seeded into 6-well. After 24 h, viral supernatants derived from Plat-E cultures were collected to infect MEFs. Two rounds of infection were performed during a $48 \mathrm{~h}$ period. Infected MEFs were subsequently cultured in mouse ES cell media and treated with ADH-1 $(5 \mu \mathrm{M})$ or ADH-AuNPs (containing $5 \mu \mathrm{M}$ ADH-1) for 4 days. After the treatment, cells were cultured in mouse ES cell media.

\section{qRT-PCR}

qRT-PCR analyses were performed with SYBR Green PCR Master Mix. Data were normalized to GAPDH expression. All analyses were performed using the ABI PRISM ${ }^{\circledR 7900 H T ~ S e q u e n c e ~ D e t e c t i o n ~ S y s t e m, ~ a n d ~ t h e ~ c o m p a r a t i v e ~ d e l t a ~ C T ~ m e t h o d ~}$ was used to calculate relative expression. The primer sequences are listed in Table S2.

\section{MTT assay}

MEF cells were seeded in 96 -well plates $\left(5 \times 10^{3}\right.$ cells/well) for $24 \mathrm{~h}$, followed by treating with either ADH-1 $(1,5,10,50,100 \mu \mathrm{M})$ or ADH-AuNPs containing different doses of ADH-1 $(1,5,10,50,100 \mu \mathrm{M})$. After 48-h incubation, MTT (20 $\mu \mathrm{L}$, $5 \mathrm{mg} / \mathrm{mL}$ ) was added to the medium for further 4-h incubation, and the medium was removed, followed by adding DMSO $(150 \mu \mathrm{L})$. The absorbance of individual well 
was measured at $570 \mathrm{~nm}$.

\section{Immunofluorescence staining}

iPS cells were fixed with 4\% paraformaldehyde for 20 min at room temperature, and permeated with $0.1 \%$ Triton X-100 for 30 min. Then, cells were blocked with $3 \%$ bovine serum albumin for 1 hour at room temperature. Afterward, cells were incubated with primary antibody (SSEA1, 1:200) in $4^{\circ} \mathrm{C}$ overnight, and then incubated with Alexa Fluor 594-conjugated goat anti-mouse secondary antibody for $1 \mathrm{~h}$ at room temperature. Furthermore, the nucleus was stained with Hoechst 33342. The cells were then washed with PBS and the images were taken by fluorescence microscopy.

\section{Quantification of reprogramming efficiency}

Two methods were used to quantify reprogramming efficiency. At about two weeks post-infection, colony numbers were counted directly under a fluorescent microscope, and then stained with alkaline phosphatase.

\section{In Vitro Differentiation of iPS Cells}

Embryoid body were generated by the hanging drop method ${ }^{1}$. In brief, iPS cells (600 cells) suspended in $20 \mu \mathrm{L}$ of ES medium without LIF were dropped onto the bottom of the lid. $10 \mathrm{~mL}$ of PBS was placed in the bottom of the dish. The lid was then inverted and incubated in the environment of $37^{\circ} \mathrm{C}, 5 \% \mathrm{CO}_{2}$ and $95 \%$ humidity. After 3 days, aggregated cells were plated in gelatin-coated culture dishes and incubated for another 7 days.

\section{Teratoma Formation}

iPS cells $\left(1 \times 10^{6}\right.$ cells $)$ were injected subcutaneously into NOD-SCID mice. Four weeks after the injection, tumors were dissected from the mice. Samples were fixed in $4 \%$ formaldehyde, and processed for paraffin embedding. Sections were stained with hematoxylin and eosin.

\section{Bisulfite Genomic Sequencing}

Genomic DNA was purified according to manufacturer's protocol and was used for bisulfite modification. The promoter regions of Nanog were amplified by PCR, and PCR primers were listed in Table S3. The amplified products were cloned into the pMD18-T vector, and ten randomly selected clones were sequenced. 


\section{Preparation and characterization of ADH-AuNPs}

Gold nanoparticles (AuNPs) were synthesized from $\mathrm{HAuCl}_{4}$ by a classic reduction method using sodium citrate as previously described ${ }^{2}$. Briefly, $100 \mathrm{~mL}$ of aqueous solution containing $0.006 \mathrm{wt} \% \mathrm{HAuCl}_{4}$ was heated to boil for $5 \mathrm{~min}$ on a hot plate under vigorous stirring, followed by adding $3 \mathrm{~mL}$ of $1 \mathrm{wt} \%$ sodium citrate solution rapidly. The reaction mixture was continuously boiled for another $30 \mathrm{~min}$ until the solution changed into wine red and then the reaction was terminated by cooling the reaction system down to room temperature to obtain AuNPs. Afterwards, AuNPs modified with ADH-1 were prepared by mixing $5 \mathrm{nM}$ AuNPs and a peptide stock solution in a volume ratio of $1: 1$, and then the reaction mixture was then kept under stirring overnight. Finally, the suspension was purified by repetitive ultrafiltration centrifugation (10000 $\mathrm{rpm}$ for $10 \mathrm{~min}$ ) to remove the unreacted $\mathrm{ADH}-1$, and then resuspended in PBS buffer to obtain ADH-AuNPs. The percentage of the loading amount of ADH-1 was determined with high performance liquid chromatography3: Loading efficacy $(\%)=\mathrm{W}_{1} / \mathrm{W}_{2} \times 100 \%$, Loading rate $(\%)=\mathrm{W}_{1} / \mathrm{W}_{3} \times 100 \%$, where $\mathrm{W}_{1}$ represents the amount of ADH-1 in ADH-AuNPs, $\mathrm{W}_{2}$ represents the initial amount of adding $\mathrm{ADH}-1, \mathrm{~W}_{3}$ represents the total amount of $\mathrm{ADH}-\mathrm{AuNPs}$.

The physical properties of ADH-AuNPs including the particle size distribution and zeta potential were measured using Zetasizer Nano ZS. The morphology of ADH-AuNPs was measured by SEM. Serum stability of ADH-AuNPs was monitored by measuring loading amount of $\mathrm{ADH}-1$ in $\mathrm{ADH}-\mathrm{AuNPs}$ at different time points. ADH-AuNPs suspension was mixed with equal volume of FBS (filtered by a $0.22 \mu \mathrm{m}$ millipore filter membrane) and incubated for $48 \mathrm{~h}$ at $37^{\circ} \mathrm{C}$. The dissociation of ADH-1 was measured at different time points to determine the stability of ADH-AuNPs.

\section{Specific binding of ADH-AuNPs on cell surface N-cadherin}

MEFs were incubated with Cy5 labeled ADH-AuNPs (Cy5/ADH-AuNPs) for $1 \mathrm{~h}$, and washed twice with PBS, imaged with confocal laser scanning microscope $(\mathrm{Ex} / \mathrm{Em}=$ 650/670 nm). In addition, MEFs were pre-incubated with $\mathrm{N}$-cadherin monoclonal antibody for $1 \mathrm{~h}$, and then treated with $\mathrm{Cy} 5 / \mathrm{ADH}-\mathrm{AuNPs}$, followed by washing twice with PBS and imaging with confocal laser scanning microscope $(\mathrm{Ex} / \mathrm{Em}=650 / 670$ 
$\mathrm{nm})$.

\section{ADH-AuNPs inhibition of N-cadh-Fc binding to MEF cells by ELISA assay}

Prior ELISA, confluent cells were detached using EDTA to protect cadherins from proteolysis and washed twice with PBS without calcium to prevent cell-cell adhesion. MEF cells were suspended in PBS containing $1.2 \mathrm{mM} \mathrm{CaCl}_{2}$ and varying concentrations $(5,10$ and $50 \mu \mathrm{M})$ of ADH-1 or ADH-AuNPs as above. After $1 \mathrm{~h}$ incubation at $37^{\circ} \mathrm{C}$, cells were added to cadherin-coated plates and incubated for further $2 \mathrm{~h}$ at $37^{\circ} \mathrm{C}$. 96 well plates were coated overnight with $30 \mathrm{ng} / \mathrm{well}$ of $\mathrm{N}$-cadherin-Fc chimeric protein or human IgG1 Fc fragment. After incubation, the wells were washed three times to remove unbound cells. The adherent cells were detected by crystal violet substrate. The intensity of the color was measured at 450 nM. Binding to human Fe fragment of IGg1 was evaluated to exclude unspecific cell binding and subtracted from those obtained with N-cadherin-Fc proteins.

\section{Statistics}

All values are reported as the mean \pm s.d. from three independent experiments. Statistical analysis was done with GraphPad Prism 6 software. Statistical significance was calculated using two-tailed Student's t-test. * $p<0.05, * * p<0.01$, and $* * * p<$ 0.001 . 


\section{Supplementary Figures}

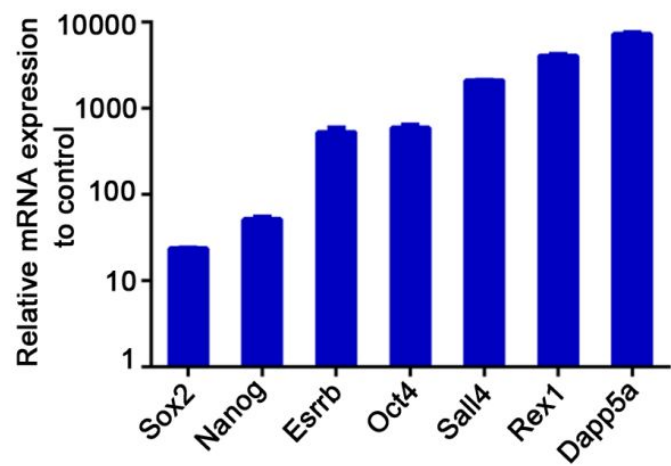

Figure S1 The expression of pluripotency marker in MEFs and iPSCs detected by qPCR.
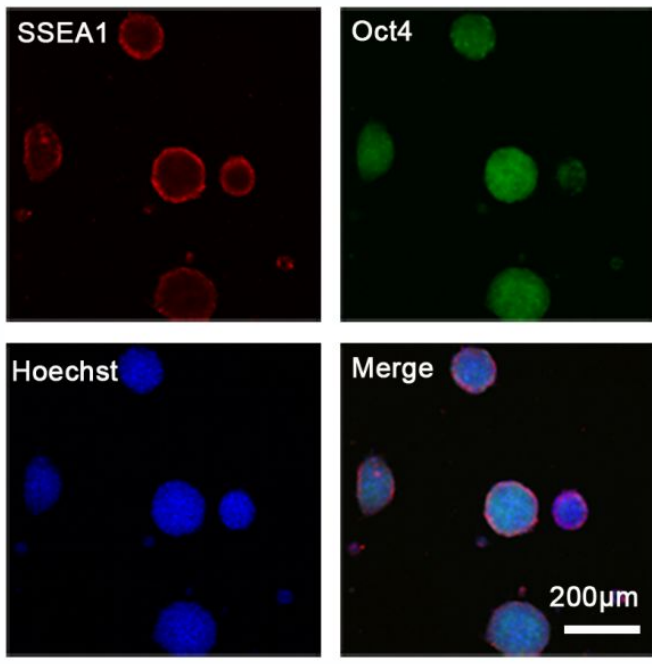

Figure S2 Pluripotency marker expression in iPSCs analyzed by immunofluorescence. Scale bar $=200 \mu \mathrm{m}$.

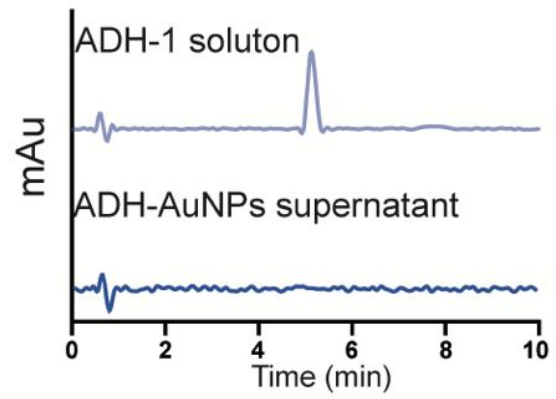

Figure S3 The concentration of ADH-1 in ADH-AuNPs supernatant detected by HPLC. 

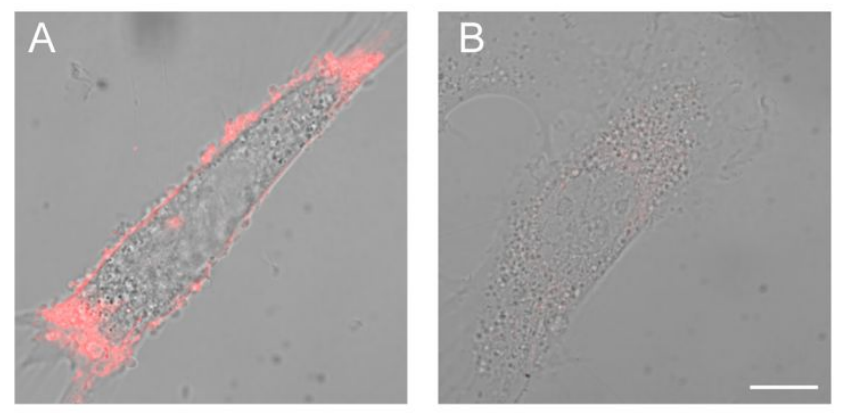

Figure S4. Specific binding of ADH-AuNPs on cell surface N-cadherin. (A) Interaction between ADH-AuNPs and cell surface N-cadherin. MEFs were incubated with Cy5 labeled ADH-AuNPs (Cy5/ADH-AuNPs) for $1 \mathrm{~h}$, and imaged with confocal laser scanning microscope. (B) Inhibition of interaction between ADH-AuNPs and cell surface N-cadherin. MEFs were pre-incubated with N-cadherin monoclonal antibody for 1h, and then treated with Cy5/ADH-AuNPs, followed by imaging with confocal laser scanning microscope. $\mathrm{Ex} / \mathrm{Em}=650 / 670 \mathrm{~nm}$, Scale bar $=10 \mu \mathrm{m}$.

\section{Supplementary tables}

Table 1 The characterization of AuNPs or ADH-AuNPs.

\begin{tabular}{lcccc}
\hline Formulations & Size $(\mathrm{nm})$ & Zeta potential $(\mathrm{mV})$ & Loading efficiacy & Loading rate \\
\hline AuNPs & $15.67 \pm 0.21$ & $-38.21 \pm 2.18$ & - & - \\
ADH-AuNPs & $20.72 \pm 0.53$ & $-25.14 \pm 1.67$ & $88.34 \pm 1.73 \%$ & $0.04 \pm 0.002 \%$ \\
\hline
\end{tabular}

Table S2 Primers sequences for qRT-PCR.

\begin{tabular}{lll}
\hline Gene name & Forward primer & Reverse primer \\
\hline Oct4 & CATTGAGAACCGTGTGAG & TGAGTGATCTGCTGTAGG \\
Rex1 & CAGCCAGACCACCATCTGTC & GTCTCCGATTTGCATATCTCCTG \\
Dapp5a & CTGGTTCCTTGGCAGGATGAT & GACATTCGAGATCCCTGTGGG \\
Nanog & CTCAAGTCCTGAGGCTGACA & TGAAACCTGTCCTTGAGTGC \\
Sox2 & AGGGCTGGGAGAAAGAAGAG & CCGCGATTGTTGTGATTAGT \\
Sall4 & CTAAGGAGGAAGAGGAGAG & CAAGGCTATGGTCACAAG
\end{tabular}




$\begin{array}{lll}\text { Esrrb } & \text { TTTCTGGAACCCATGGAGAG } & \text { AGCCAGCACCTCCTTCTACA } \\ \text { N-cadherin } & \text { GGAATCCCGCCTATGAGTGG } & \text { GGAAGATCAAACGCGAACGG } \\ \text { Vimentin } & \text { GGATCAGCTCACCAACGACA } & \text { AAGGTCAAGACGTGCCAGAG } \\ \text { ZEB1 } & \text { GCGGCGCAATAACGTTACAA } & \text { AATACTGTCTGGTCTGCTGGC } \\ \text { E-cadherin } & \text { ATCATTGAGAGGGAGACAGGC } & \text { CAACGGATCCCTCAAACACCTC }\end{array}$

Table S3 Primers sequences for PCR.

Forward primer Reverse primer

ATtAATtGTGAATtTATAGGgtTGG AACCCACACTCATATCAATATAATAAC

\section{Reference}

1. Boheler, K. R.; Czyz, J.; Tweedie, D.; Yang, H. T.; Anisimov, S. V.; Wobus, A.

M. Differentiation of pluripotent embryonic stem cells into cardiomyocytes. Circ Res 2002, 91, (3), 189-201.

2. Cheng, X.; Sun, R.; Yin, L.; Chai, Z.; Shi, H.; Gao, M. Light-triggered assembly of gold nanoparticles for photothermal therapy and photoacoustic imaging of tumors in vivo. Adv Mater 2017, 29, (6), 6.

3. Perotti, A.; Sessa, C.; Mancuso, A.; Noberasco, C.; Cresta, S.; Locatelli, A.; Carcangiu, M. L.; Passera, K.; Braghetti, A.; Scaramuzza, D.; Zanaboni, F.; Fasolo, A.; Capri, G.; Miani, M.; Peters, W. P.; Gianni, L. Clinical and pharmacological phase I evaluation of Exherin (ADH-1), a selective anti-N-cadherin peptide in patients with N-cadherin-expressing solid tumours. Ann Oncol 2009, 20, (4), 741-5. 